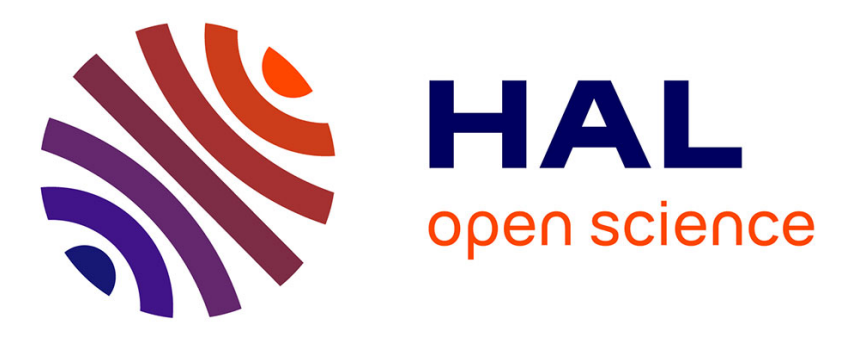

\title{
Evaluation of the Colorimetric Performance of Single-Sensor Image Acquisition Systems Employing Colour and Multispectral Filter Array
}

Xingbo Wang, Philips John Green, Jean-Baptiste Thomas, Jon Yngve Hardeberg, Pierre Gouton

\section{To cite this version:}

Xingbo Wang, Philips John Green, Jean-Baptiste Thomas, Jon Yngve Hardeberg, Pierre Gouton. Evaluation of the Colorimetric Performance of Single-Sensor Image Acquisition Systems Employing Colour and Multispectral Filter Array. The Fifth IAPR Computational Color Imaging Workshop (CCIW'15), Mar 2015, Saint Etienne, France. pp.181-191, 10.1007/978-3-319-15979-9_18 . hal01214047

\section{HAL Id: hal-01214047 \\ https://hal.science/hal-01214047}

Submitted on 15 Oct 2015

HAL is a multi-disciplinary open access archive for the deposit and dissemination of scientific research documents, whether they are published or not. The documents may come from teaching and research institutions in France or abroad, or from public or private research centers.
L'archive ouverte pluridisciplinaire HAL, est destinée au dépôt et à la diffusion de documents scientifiques de niveau recherche, publiés ou non, émanant des établissements d'enseignement et de recherche français ou étrangers, des laboratoires publics ou privés. 


\title{
Evaluation of the colorimetric performance of single-sensor image acquisition systems employing colour and multispectral filter array
}

\author{
Xingbo Wang ${ }^{1,2}$, Philip J. Green ${ }^{1}$, Jean-Baptiste Thomas ${ }^{2}$, \\ Jon Y. Hardeberg ${ }^{1}$, and Pierre Gouton ${ }^{2}$ \\ 1 Norwegian Colour and Visual Computing Laboratory \\ Gjøvik University College, Gjøvik 2815, Norway \\ xingbo . wang@hig.no \\ WWW home page: http://www.colourlab.no http://www.springer.com/lncs \\ ${ }^{2}$ Laboratoire Electronique, Informatique et Image \\ Université de Bourgogne, Dijon, France \\ WWW home page: http://le2i.cnrs.fr
}

\begin{abstract}
Single-sensor colour imaging systems mostly employ a colour filter array (CFA). This enables the acquisition of a colour image by a single sensor at one exposure at the cost of reduced spatial resolution. The idea of CFA fit itself well with multispectral purposes by incorporating more than three types of filters into the array which results in multispectral filter array (MSFA). In comparison with a CFA, an MSFA trades spatial resolution for spectral resolution. A simulation was performed to evaluate the colorimetric performance of such CFA/MSFA imaging systems and investigate the trade-off between spatial resolution and spectral resolution by comparing CFA and MSFA systems utilising various filter characteristics and demosaicking methods including intraand inter-channel bilinear interpolation as well as discrete wavelet transformed based techniques. In general, 4-band and 8-band MSFAs provide better or comparable performance than the CFA setup in terms of CIEDE2000 and S-CIELAB colour difference. This indicates that MSFA would be favourable for colorimetric purposes.
\end{abstract}

Keywords: colorimetric performance, colour filter array, multispectral imaging, single-sensor

\section{Introduction}

Single-sensor trichromatic imaging systems mostly employ a colour filter array (CFA) in order to sense a portion of the incoming spectra selectively on a pixel-by-pixel basis. An example of CFA that has achieved commercially notable success is known as Bayer filter mosaic consisting of three types of filters, i.e., red, blue and green [2], as shown in Figure 1. Thanks to the spatial and spectral inter-pixel correlation an image may possess, the lost information about the incident stimuli can be estimated through demosaicking. Demosaicking is 
an operation in the image processing chain carried out on the mosaicked image read from the sensor. Consequently each pixel will comprise three components, thereby recovering a full colour image. The success gained by the CFA based single-sensor colour imaging systems has awakened particular interest from the academia and the industries in generalise this concept to the multispectral domain by integrating more than three types of filters into one filter array, which results in the multispectral filter array (MSFA). Two instances of MSFAs can be seen in Figure 2 and 3.

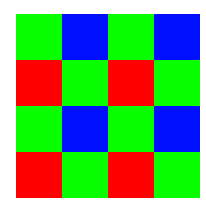

Fig. 1. Bayer CFA.

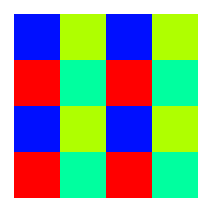

Fig. 2. 4-band MSFA.

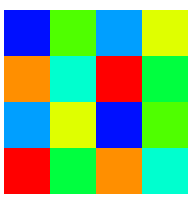

Fig. 3. 8-band MSFA.

In general, the development of a MSFA based imaging system involves design of filter transmittances, spatial arrangement of mosaic patterns paired with an associated demosaicking algorithm, and a regression process to recover colorimetric or spectral information.

In recent years, sustained research effort went into designing multispectral filter array (MSFA) [15] and developing associated demosaicking algorithms [14, $1,3,16,17,21,22]$. Also widely explored is the influence of filter characteristics on colour/spectrum reproduction [19, 9, 23, 24]. Nevertheless, to our best knowledge, little is known about how filter design and demosaicking algorithms affect the colorimetric performance of a CFA and particularly MSFA image acquisition system.

In comparison with CFAs, MSFAs populate higher number of channels, thus reducing the number of pixels assigned to a certain channel for a given sensor. Obviously this lowers spatial resolution, however MSFA may offer higher spectral resolution. While the former effect generally lowers the colorimetric performance of the system, the latter should improve the accuracy of colour reproduction. It is therefore of particular interest to evaluate the colorimetric performance of such MSFA imaging systems and investigate the trade-off between spatial resolution and spectral resolution by comparing CFA and MSFA systems utilising various filter characteristics and demosaicking methods.

The following sections of the paper are organised as follows. We first present the methods used including the simulated framework illustrated in Section 2.1, the filter design strategy described in Section 2.2, the mosaic generation demonstrated in Section 2.3, the demosaicking algorithms introduced in Section 2.4, the colour and spectral reflectance estimation method for device calibration presented in Section 2.5, the evaluation means explained in Section 2.6 and the 
experimental conditions listed in Section 2.7. Results are shown in Section 3 that leads to reasonable conclusions drawn in Section 4.

\section{Methods}

\subsection{Simulated framework}

A simulated workflow was constructed so as to conduct the research due to practical difficulties in physical implementation of the MSFAs [12]. As shown in Figure 4, the framework consists of a chain of processing that starts from the hyperspectral images used as virtual optical images. A MSFA mounted sensor is merely a combination of the filter array and the image sensor, and the mosaicked optical image is therefore formed in between. As a result, a hyperspectral image can be considered as a spectrally sampled optical image which will then be spectrally filtered and spatially interleaved by the filter array. In this manner, the process of mosaicking is simulated.

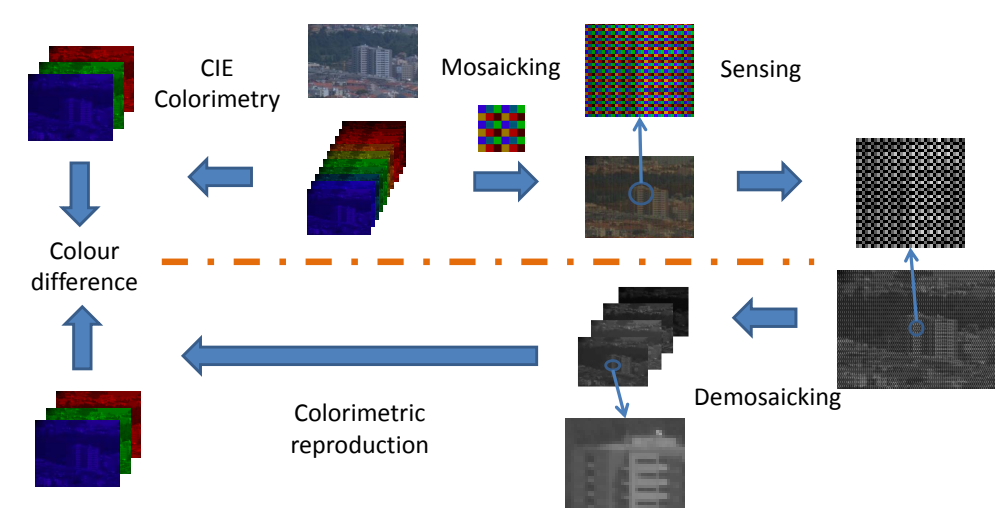

Fig. 4. The experimental framework.

Next, an ideal image sensor populating the same number of pixels as the images integrates the incident power at each pixel over the spectrum. Quantum efficiency of the sensor is integrated with the spectral transmittance of filters, so that filter characteristics referred to in this work represent sensor sensitivities. And neither optical crosstalk nor optical/electronic noise is considered in this work.

Mosaic image, namely the sensor output, is actually a digital representation of a spatially or spectrally subsampled and interleaved trichromatic or multispectral image. Therefore, it needs to be interpolated spatially and/or spectrally, in order to recover the information lost in the mosaicking process. In other words, interpolation yields a colour or multispectral image of full spatial resolution. 
At this stage, the recovered image is not colorimetrically meaningful as the digital counts have not been assigned any physical meaning. This is addressed by device colour calibration. It first models the device by associating stimuli with known colorimetric characteristics and the corresponding sensor response, and later estimates original colorimetric information of the unknown stimuli from the corresponding sensor response.

CIE tristimulus values are computed before colour difference between the original and reproduced hyperspectral images is calculated.

\subsection{Filter design in MSFA systems}

Among the derivatives of Bayer mosaic, some possess complementary colour filters in comparison to the primary colour filters utilised in the original patent [2].

Literature presents distinct results. It is obvious that complementary colour filters intrinsically bear wider pass-band than their primary counterparts, and it is widely accepted that the former gives rise to better colour reproduction and signal-to-noise ratio in sufficient lighting conditions, whereas the latter offers higher sensitivity as well as resolution $[19,18]$. Our results, nevertheless, show that appropriate pass-bands outperforms some narrower ones [23,24]. In addition, our previous research on multispectral demosaicking poses the question of filter design in relation to the inter-band correlation $[21,22]$.
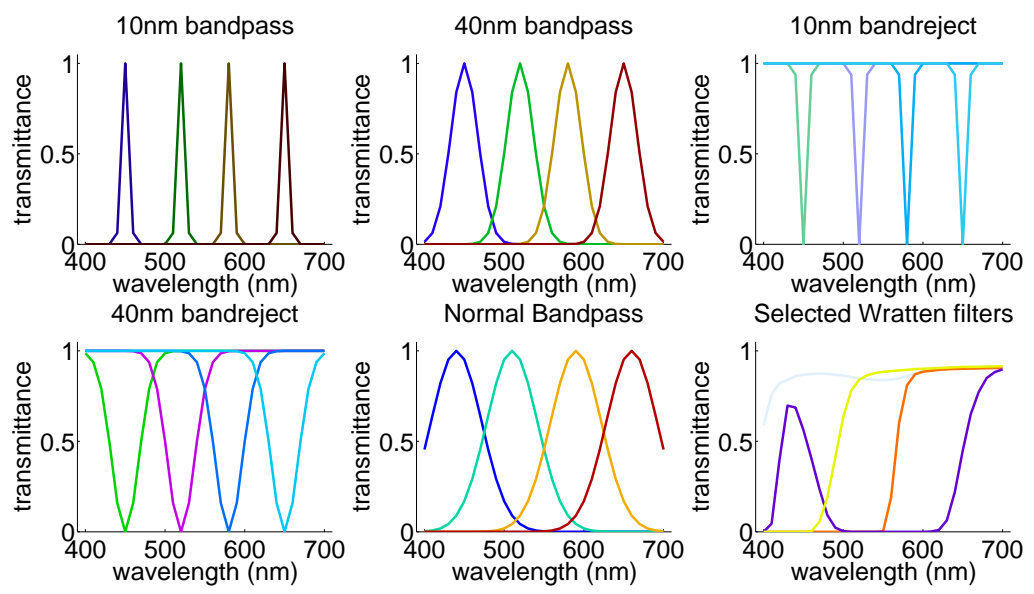

Fig. 5. Transmittances of a 4-band filter set.

An instance of 4-band filter set used in this research is depicted in Figure 5. Following the aforementioned findings, we are interested in narrowband and broadband bandpass filters as well as corresponding inverted ones such as bandstop filters. The FWHM (Full Width at Half Maximum) of passband and 
stopband have been set to $10 \mathrm{~nm}$ and $40 \mathrm{~nm}$ respectively. In practice, a passband of $10 \mathrm{~nm}$ simulates very narrow bandpass filters like LCTF (Liquid Crystal Tunable Filter), a stopband of $10 \mathrm{~nm}$ mimics notch filters relying on destructive interference. Similarly, a passband and a stopband of $40 \mathrm{~nm}$ resemble the spectral transmittances of thin-film filters.

In addition to the filters mentioned above, we introduced two more types. One is based on the principle that the transmittances of filters should sample the spectrum evenly with their FWHM. The other is in fact the result produced by a filter selection algorithm [7] that chooses a given number of optimal(or suboptimal) filters from a set of available candidates that are physically practicable, on the assumption that high spectral performance is yielded by the "brightest" filter that transmits the most light combined with other filters which are orthogonal to each other in a vector space. Here we employ a set of transmittance data measured from Wratten filters produced by Kodak, as shown in Figure 6 .

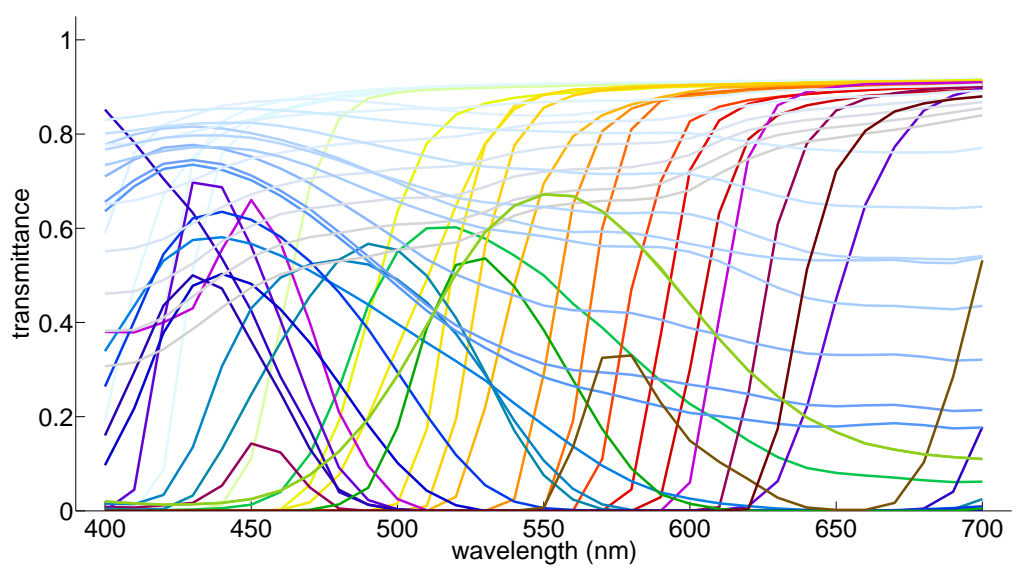

Fig. 6. Transmittances of a set of Wratten filters.

\subsection{Mosaic pattern generation}

Filter arrays experimented with in this project were designed with the help of a generic binary tree based generation method of MSFA spatial arrangement starting from a chequerboard pattern introduced by Miao et al. [15]. By manipulating the pattern through a combination of decomposition and subsampling steps, the method presented may generate MSFAs that satisfy varied design requirements proposed by the authors including probability of appearance, spectral consistency and uniform distribution. It is shown, through case studies, that most of the CFAs currently used by the industry can be derived as special cases. 


\subsection{MSFA demosaicking}

A large number of CFA demosaicking algorithms have been proposed over the decades [13]. In this work, we used intra-channel bilinear interpolation, channel difference bilinear interpolation [11] and discrete wavelet transform (DWT) based demosaicking [5].

Bilinear interpolation makes use of merely intra-pixel correlation and estimates unknown colour components by exploiting the spatial correlation between sampled colours in a certain spectral plane, and function plane by plane.

Channel difference interpolation brings inter-channel correlation into play and interpolates the difference between one colour plane and another [11] on the assumption that hue changes smoothly in images.

DWT transforms an image into various frequency bands, and natural images often possess rather similar high-frequency information among these bands, which provides yet another solution to demosaicking problem. This algorithm has been extended to a 4-band MSFA as reported in the literature [22].

\subsection{Device calibration}

Colour calibration is an inverse problem aimed at an estimation of the tristimulus values of the stimuli from the corresponding measurements obtained from sensors. In concrete terms, a colour acquisition process can be described in a linear form as

$$
R=Q S
$$

where $R$ refers to the sensor responses, $Q$ corresponds to system responsivities and $S$ represents the incoming stimuli.

Colour calibration aims at an estimation of $S$ from $R$. Equation 1 is solvable if $Q$ is known and invertible, however it is not true in the case of colorimetric calibration. Nevertheless it can be estimated by means of training where a collection of training stimuli $S_{t}$ and corresponding responses $R_{t}$ are utilised to derive an approximation of $Q^{-1}$. In this work, we employed the method of linear least squares [8] which attempts to solve (1) by means of pseudoinverse which leads to $(2)$

$$
S^{\prime}=S_{t} R_{t}^{+} R
$$

where $S^{\prime}$ is an estimation of $S$ and $R_{t}^{+}$is a right pseudoinverse of $R_{t}: R_{t}^{+}=$ $R_{t}^{T}\left(R_{t} R_{t}^{T}\right)^{-1}$.

\subsection{Performance evaluation}

An evaluation of the colorimetric performance of CFA and MSFA based imaging systems can be solved by means of colour difference formula. However, considering that the targets are digital images rather than uniform colour patches, a 
metric incorporating some low-level HVS features, such as S-CIELAB, might be suitable as well and may provide more information.

CIEDE2000 is the latest colour difference formula developed and recommended by the CIE [4]. It further improves perceptual uniformity in comparison with the CIE94 formula by introducing a few revised compensation terms for lightness, chroma and hue respectively. In addition, there are three corresponding parameters that are usually set to 1:1:1 and can be adjusted according to specific applications. For instance, CIE recommended 2:1:1 for textile industry. In this work, we used 2:2:1 to evaluate image colour difference.

The S-CIELAB metric extends the CIELAB Delta E metric to colour images by adding a spatial pre-processing step to the standard CIE $\Delta E_{a^{*} b^{*}}$ metric to account for the spatial-colour sensitivity of the human eye [26]. It measures how accurate the reproduction of a colour is to the original when viewed by a human observer.

\subsection{Experimental conditions}

The experiments were conducted in such conditions as follows. Spectral range covers the spectrum between $400 \mathrm{~nm}$ and $700 \mathrm{~nm}$ with $10 \mathrm{~nm}$ interval. CIE D65 was used as the illuminant. Among the 48 hyperspectral images used as virtual scenes, 16 are from Foster database [6] and 32 are from CAVE database [25]. Three types of MSFA were considered, namely 3-band CFA, 4-band and 8-band. For the least-square regression, 170 spectral reflectances of natural objects [20] and the corresponding CIE XYZ tristimulus values were utilised as the training targets. Tristimulus values were calculated with colour-matching functions for the CIE 1931 standard colorimetric observer [10]. For the calculation of SCIELAB colour difference, the viewing distance was set to $60 \mathrm{~cm}$, and the resolution was set to $95.78 \mathrm{dpi}$, so as to mimic a 23-inch LCD monitor of $1920 \times 1080$ pixels and an aspect ratio of 16:9.

\section{Results and discussion}

Results are presented in Figure 7 and 8. It is of great moment to realise that the colour difference shown here reflect the overall performance of the system consisting of filter characteristics, spatial arrangements, demosaicking methods as well as colour estimation techniques. However, a comparative analysis of the results reveal some clues.

From the results we can observe that increased number of bands in general offer lower or comparable colour difference especially when paired with $10 \mathrm{~nm}$ and $40 \mathrm{~nm}$ bandpass filters and a selected range of Wratten filters. In particular, the $40 \mathrm{~nm}$ bandpass filters result in the lowest colour difference among all of the methods and configurations, whereas the $10 \mathrm{~nm}$ bandstop filters yield significantly larger errors.

In general, the DWT based demosaicking outperforms the other two where the widths of passband are significantly broader, whereas bilinear interpolation 


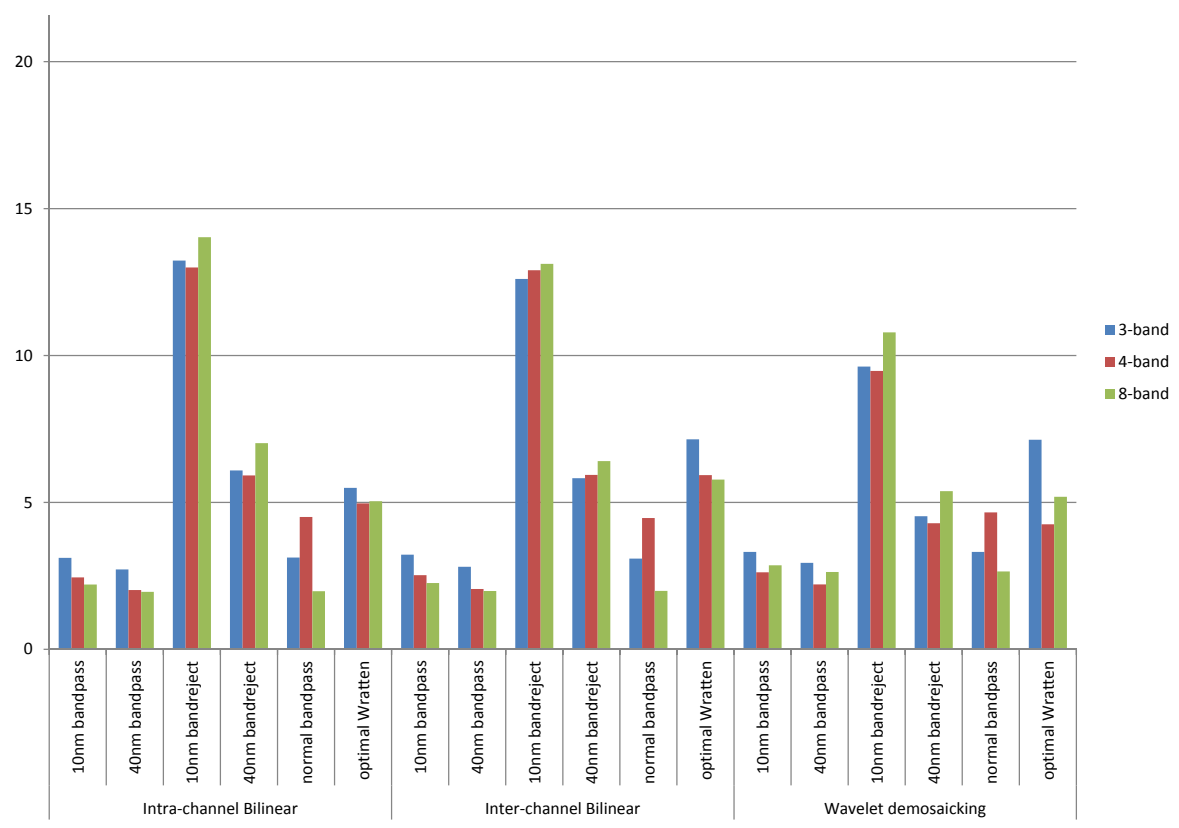

Fig. 7. Average CIEDE2000 colour difference among test images.

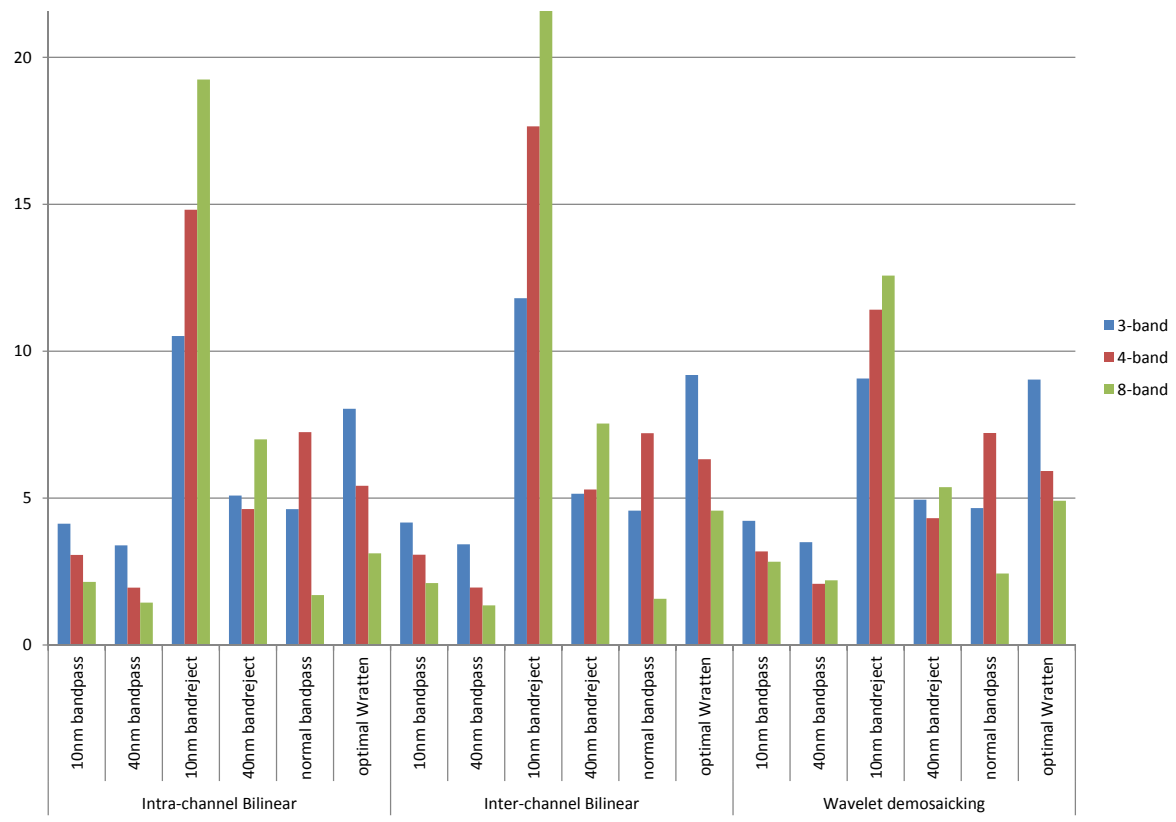

Fig. 8. Average S-CIELAB colour difference among test imags. 
carried out on channel differences does not perform satisfactorily. This is also related to insufficient high-frequency components in the image databases [22], as inter-channel demosaicking should benefit from inter-channel correlation at high frequencies and broader passbands may boost this correlation.

In most cases S-CIELAB results coincide with CIE DE2000 ones, although the former tends to exaggerate the discrepancy of the results between the CFA and the MSFAs.

\section{Conclusion}

A simulation was performed to investigate the colorimetric performance of MSFA based image acquisition systems. In total, 48 virtual scenes were captured by the simulated camera for 3-band, 4-band and 8-band MSFAs respectively, each paired with 6 different types of filter characteristics and 3 demosaicking algorithms. Results were all transformed to CIE XYZ tristimulus values and evaluated with CIEDE2000 (2:2:1) and S-CIELAB colour difference. CIEDE2000 and $\mathrm{S}$-CIELAB results coincide in most cases. In general, the 4-band MSFA provides better or comparable performance in comparison with the 3-band setup except the case of $10 \mathrm{~nm}$ bandreject and normal bandpass filters. Similarly the 8-band MSFA delivers higher colour accuracy expect the case of $10 \mathrm{~nm}$ and $40 \mathrm{~nm}$ bandreject filters. Therefore MSFA is generaly helpful for an application where colorimetric reproduction is required.

Moreover, it is obvious to see that spectral characteristics of a filter set not only make a direct impact on the colour reconstruction, but also influence the spectral correlation of the observed image on which some demosaicking methods depend.

Certainly the validity of the results obtained in this work is limited by the realisticness of the simulation. In a real system, the presence of various types of noise will probably impact the results. However, this laid a foundation for the design of a new MSFA sensor planned in 2015 [12].

\section{References}

1. Baone, G.A., Qi, H.: Demosaicking methods for multispectral cameras using mosaic focal plane array technology. In: Proc. SPIE. vol. 6062 (Jan 2006)

2. Bayer, B.E.: Color imaging array. Patent (07 1976), uS 3971065

3. Brauers, J., Aach, T.: A color filter array based multispectral camera. In: Group, G.C. (ed.) 12th Workshop Farbbildverarbeitung. Ilmenau (October 5-6 2006)

4. Colorimetry - Part 6: CIEDE2000 Colour-Difference Formula. No. CIE Draft Standard DS 014-6/E:2012, CIE, Vienna, Austria (2012)

5. Driesen, J., Scheunders, P.: Wavelet-based color filter array demosaicking. In: International Conference on Image Processing (ICIP). vol. 5, pp. 3311-3314. IEEE (Oct 2004)

6. Foster, D.H., Amano, K., Nascimento, S.M.C., Foster, M.J.: Frequency of metamerism in natural scenes. J. Opt. Soc. Am. A 23(10), 2359-2372 (Oct 2006) 
7. Hardeberg, J.Y.: Acquisition and Reproduction of Color Images: Colorimetric and Multispectral Approaches. dissertation.com (2001)

8. Hironaga, M., Shimano, N.: Estimating the noise variance in an image acquisition system and its influence on the accuracy of recovered spectral reflectances. Appl. Opt. 49(31), 6140-6148 (Nov 2010)

9. Imai, F.H., Rosen, M.R., Berns, R.S.: Comparison of spectrally narrow-band capture versus wide-band with a priori sample analysis for spectral reflectance estimation. In: Proceedings of the IS\&T/SID Eighth Color Imaging Conference: Color Science and Engineering Systems, Technologies, and Applications. pp. 234-241. IS\&T, Scottsdale, Arizona (Nov 2000)

10. CIE Colorimetry - Part 1: Standard Colorimetric Observers. No. ISO 116641:2007(E)/CIE S 014-1/E:2006, ISO, Geneva, Switzerland (2007)

11. James E. Adams, J.: Interactions between color plane interpolation and other image processing functions in electronic photography. In: SPIE. vol. 2416, pp. 144-151 (1995)

12. Lapray, P.J., Wang, X., Thomas, J.B., Gouton, P.: Multispectral filter arrays: Recent advances and practical implementation. Sensors 14(11), 21626-21659 (2014)

13. Menon, D., Calvagno, G.: Color image demosaicking: An overview. Image Commun. 26(8-9), 518-533 (Oct 2011)

14. Miao, L., Qi, H., Ramanath, R.: A generic binary tree-based progressive demosaicking method for multispectral filter array. In: IEEE International Conference on Image Processing. pp. 3221 -3224 (Oct 2006)

15. Miao, L., Qi, H., Snyder, W.E.: A generic method for generating multispectral filter arrays. In: International Conference on Image Processing. vol. 5, pp. $3343-$ 3346 (Oct 2004)

16. Monno, Y., Tanaka, M., Okutomi, M.: Multispectral demosaicking using adaptive kernel upsampling. In: 18th IEEE International Conference on Image Processing (ICIP). pp. 3157-3160. Brussels (Sep 2011)

17. Monno, Y., Tanakaa, M., Okutomia, M.: Multispectral demosaicking using guided filter. In: Battiato, S., Rodricks, B.G., Sampat, N., Imai, F.H., Xiao, F. (eds.) Digital Photography VIII. Proc. of SPIE, vol. 8299, pp. 82990O-82990O-7. Burlingame, California, USA (Jan 2012)

18. Panasonic: Primary color and complementary color filters. http://panasonic.jp/ support/global/cs/dsc/knowhow/knowhow29.html (retrieved on Dec 16, 2013)

19. Parulski, K.A.: Color filters and processing alternatives for one-chip cameras. IEEE Transactions on Electron Devices 32(8), 1381-1389 (1985)

20. Vrhel, M.J., Gershon, R., Iwan, L.S.: Measurement and analysis of object reflectance spectra. Color Research \& Application 19(1), 4-9 (1994)

21. Wang, X., Thomas, J.B., Hardeberg, J.Y., Gouton, P.: Median filtering in multispectral filter array demosaicking. In: Digital Photography IX. Proc. SPIE, vol. 8660

22. Wang, X., Thomas, J.B., Hardeberg, J.Y., Gouton, P.: Discrete wavelet transform based multispectral filter array demosaicking. In: Proc. Colour and Visual Computing Symposium (CVCS), 2013. pp. 1-6 (Sep 2013)

23. Wang, X., Thomas, J.B., Hardeberg, J.Y., Gouton, P.: A study on the impact of spectral characteristics of filters on multispectral image acquisition. In: Proc. $12^{\text {th }}$ AIC Congress. AIC (Jul 2013)

24. Wang, X., Thomas, J.B., Hardeberg, J.Y., Gouton, P.: Multispectral imaging: narrow or wide band filters? Journal of the International Colour Association 12 (Jul 2014) 
25. Yasuma, F., Mitsunaga, T., Iso, D., Nayar, S.K.: Generalized assorted pixel camera: Post-capture control of resolution, dynamic range and spectrum. Tech. rep., Department of Computer Science, Columbia University CUCS-061-08 (Nov 2008), http://www1.cs.columbia.edu/CAVE/databases/multispectral/

26. Zhang, X., Wandell, B.A.: A spatial extension of cielab for digital color-image reproduction. Journal of the Society for Information Display 5(1), 61-63 (1997) 